Meta

Journal des traducteurs

Translators' Journal

\title{
Les postes de soudage à l'arc
}

\section{Geneviève Henry}

Volume 33, numéro 3, septembre 1988

URI : https://id.erudit.org/iderudit/003060ar

DOI : https://doi.org/10.7202/003060ar

Aller au sommaire du numéro

Éditeur(s)

Les Presses de l'Université de Montréal

ISSN

0026-0452 (imprimé)

1492-1421 (numérique)

Découvrir la revue

Citer cet article

Henry, G. (1988). Les postes de soudage à l'arc. Meta, 33(3), 407-416.

https://doi.org/10.7202/003060ar

Ce document est protégé par la loi sur le droit d'auteur. L'utilisation des services d'Érudit (y compris la reproduction) est assujettie à sa politique d'utilisation que vous pouvez consulter en ligne.

https://apropos.erudit.org/fr/usagers/politique-dutilisation/
Cet article est diffusé et préservé par Érudit.

Érudit est un consortium interuniversitaire sans but lucratif composé de l’Université de Montréal, l'Université Laval et l'Université du Québec à Montréal. Il a pour mission la promotion et la valorisation de la recherche. https://www.erudit.org/fr/ 


\section{LES POSTES DE SOUDAGE À L'ARC*}

Le poste de soudage ${ }^{1}$, également appelé appareil de soudage, ou source de courant de soudage (welding power source ${ }^{2}$ ), constitue, dans tous les procédés de soudage électrique, la pierre angulaire du matériel.

Par rapport aux postes de soudage, les postes de soudage à l'arc (arc-welding power source; arc welding power source; arc welding power supply ${ }^{3}$ ) constituent un type d'appareil de soudage ; autrement dit, ce terme constitue un spécifique par rapport à " poste de soudage ". Même s'ils diffèrent d'un procédé à un autre (soudage TIG, soudage MIG, soudage au plasma, etc.), les postes de soudage à l'arc ont tous pour rôle de permettre l'amorçage et le maintien d'un arc stable, de fournir un débit de courant satisfaisant sans donner lieu, en service, à des incidents pouvant nuire au soudage ou au fonctionnement de l'installation. Leur classification peut s'effectuer suivant plusieurs lignes directrices. Certains auteurs se fondent par exemple sur les caractéristiques du courant nécessaires au bon fonctionnement d'un procédé. On distingue ainsi des postes de soudage produisant un courant constant (constant current) et d'autres produisant une tension constante (constant voltage).

D'autres auteurs préfêrent diviser les postes de soudage à l'arc selon des caractéristiques mécaniques : les appareils statiques (static power source), qui ne comportent pas d'organes tournants engendrant le courant de soudage, un transformateur par exemple, par opposition aux appareils rotatifs (rotating power source), une génératrice par exemple. Finalement, la majorité des auteurs classent les postes de soudage à l'arc selon le type de courant produit. On trouve ainsi, d'une part, les appareils produisant du courant continu et, d'autre part, les appareils produisant du courant alternatif. D'un point de vue terminologique, cette classification a le mérite de mieux faire ressortir les différents niveaux hiérarchiques entre les termes. C'est également celle qui, d'un point de vue didactique, permet de présenter au lecteur le plus grand nombre d'unités terminologiques. Ces raisons nous ont fait préférer cette dernière classification (voir figure 1).

\section{LES APPAREILS À COURANT ALTERNATIF}

Ce type de poste de soudage à l'arc est utilisé dans des procédés tels que le soudage TIG pour l'aluminium, le magnésium et d'autres métaux réfractaires, car le courant alternatif prévient l'accumulation d'oxydes métalliques à la surface du bain de soudure. Les appareils à courant alternatif ou sources de courant alternatif ( $A C$ power source ; $A C$ welding power source ${ }^{4}$ ) se divisent en deux types principaux : les génératrices de courant alternatif ( $A C$ generator; $A C$ arc welding generator) et les transformateurs de courant alternatif ${ }^{5}$ [(terme non attesté en français, construit par analogie avec " génératrice de courant alternatif") (AC transformer; $A-C$ transformer $\left.\left.{ }^{6}\right)\right]$.

a) Les génératrices de courant alternatif (appareils rotatifs)

La génératrice de courant alternatif utilise l'énergie mécanique engendrée soit par un moteur électrique, soit par un moteur thermique, pour la production de tensions et 
Figure 1

GÉNÉRIQUE

SPÉCIFIQUE

TYPE DE COURANT

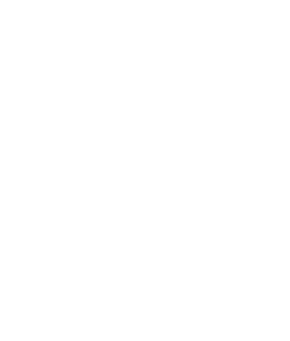

TECHNOLOGIE

a) GÉNÉRATRICE DE COURAN

*AC arc welding machine; A-C power source: AC arc-welding machine; A-C power supply;

*AC machine;

*A-C welding machine

AC power source.

AC welding power source

A-C welding power

source;

alternating-current

power source;

alternating-current

welding power source;

*alternating-current

welding machine

i

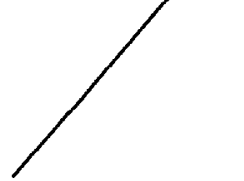

ALTERNATIF

TRANSFORMATEUR

DE COURANT

ALTERNATIF

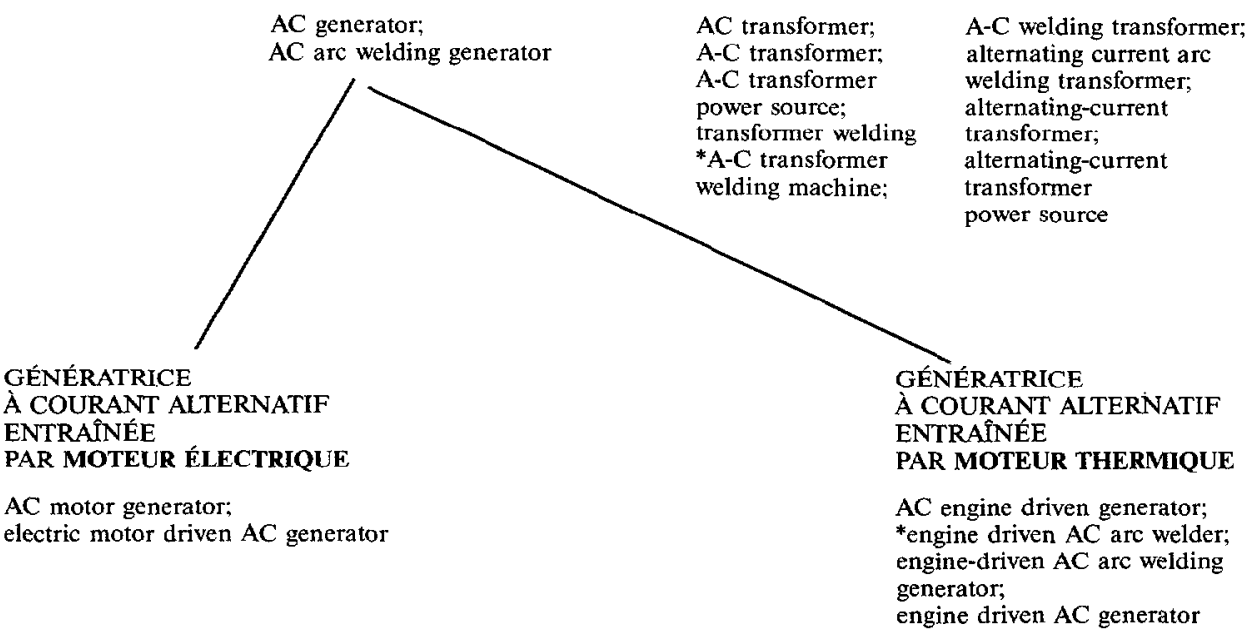


POSTE DE SOUDAGE

$\begin{array}{ll}\begin{array}{ll}\text { appareil de soudage; } \\ \text { source de courant; }\end{array} & \begin{array}{l}\text { source de courant } \\ \text { de soudage; } \\ \text { *welder; } \\ \text { *welding machine; } \\ \text { power source; }\end{array} \\ \text { power supply; } & \begin{array}{l}\text { welding power source } \\ \text { POSTE DE SOUDAGE À LARC }\end{array}\end{array}$

*arc welder;

*arc welding

machine;

are welding power source; arc-welding power source; arc welding power supply

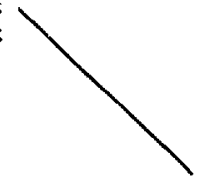

II APPAREIL À COURANT CONTINU : source de courant continu

*DC arc welder;

*DC arc welding machine;

*DC machine;

DC power source;

D-C power source;
*DC welder;

*DC welding machine

DC welding power source;

direct current machine

direct current power supply;

*direct-current welder a) GÉNÉRATRICE

DE COURANT CONTINU
DC generator; direct-current direct-current arc welding welding generator generator;

GÉNÉRATRICE

A COURANT CONTINU

\section{ENTRAÎNÉE}

\section{PAR MOTEUR ÉLECTRIQUE}

DC motor generator; * DC motor generator arc welder,
*DC motor-generator welding machine

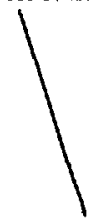

b) TRANSFORMATEUR-

REDRESSEUR:

transformateur-redresseur à courant continu

DC rectifier power source; transformer-rectifier D-C D-C transformer-rectifier; welding machine;

D-C transformer-rectifier transformer-rectifier welding power source; machine;

transformer-rectifier transformer-rectifier D-C power source; $\quad$ power source
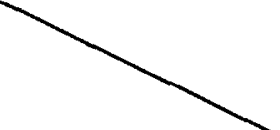

GÉNÉRATRICE

A COURANT CONTINU

ENTRAÎNÉE

PAR MOTEUR THERMIQUE

DC engine-driven power source 
de courants alternatifs monophasés ou polyphasés. Les génératrices à courant alternatif entraînées par moteur électrique ( $A C$ motor generator ; electric motor driven $A C$ generator) sont de moins en moins utilisées de nos jours. On se sert plutôt de génératrices à courant alternatif entraînées par moteur thermique ( $A C$ engine driven generator; engine-driven $A C$ arc welding generator; engine driven $A C$ generator ${ }^{7}$ ) ou de génératrices à courant continu entraînées par moteur thermique lorsque l'on soude dans des endroits où il est difficile d'avoir accès à des lignes électriques (chantiers de construction, fermes, etc.). Les moteurs des génératrices consomment généralement de l'essence ou de l'huile lourde, mais ils peuvent également être à gaz ou même à vapeur.

b) Les transformateurs de courant alternatif (appareils statiques)

Les transformateurs de courant alternatif sont les appareils de soudage les plus utilisés. L'organe principal est le transformateur de soudage (welding transformer). Son principe de fonctionnement est le même que celui du transformateur abaisseur de tension (step-down transformer) : il abaisse la tension du courant alternatif fourni par le réseau pour donner une tension et une intensité convenant au procédé en cours. Le transformateur est constitué d'un circuit magnétique et de deux enroulements, ou bobinages, l'un dit primaire (primary circuit), l'autre, secondaire (secondary circuit).

\section{LES APPAREILS À COURANT CONTINU}

Les appareils à courant continu, ou sources de courant continu ( $D C$ power source; $D C$ welding power source ${ }^{8}$, se divisent également en deux groupes principaux : les génératrices de courant continu ( $D C$ generator; direct-current arc welding generator; direct-current welding generator) et les transformateurs-redresseurs ou transformateurredresseur à courant continu ( $D C$ transformer-rectifier; $D-C$ transformer-rectifier welding power source ${ }^{9}$ ).

\section{a) Les génératrices de courant continu}

Tout comme les génératrices de courant alternatif, elles sont entraînées soit par moteur électrique, soit par moteur thermique. Ce sont, dans le premier cas, des génératrices à courant continu entraînées par moteur électrique [(terme non attesté construit par analogie avec "génératrice à courant alternatif entraînée par moteur électrique ") $\left(D C\right.$ motor generator $\left.{ }^{10}\right)$ ] et dans le second cas, des génératrices à courant continu entraînées par moteur thermique ( $D C$ engine-driven power source).

\section{b) Les transformateurs-redresseurs}

Le transformateur-redresseur est un appareil statique qui se compose d'un transformateur abaisseur de tension et d'un redresseur (rectifier). Le redresseur transforme le courant alternatif qui le traverse en un courant assimilable, du point de vue de ses propriétés, au courant continu. Il existe plusieurs types de redresseurs. Parmi les plus courants en soudage, on trouve le redresseur au silicium (silicon rectifier; silicon diode; silicon-diode rectifier; silicon-controlled rectifier; $S C R$ ) et le redresseur au sélénium (selenium rectifier; selenium diode). Certains transformateurs-redresseurs ne produisent que du courant continu. D'autres sont conçus pour être utilisés indifféremment comme transformateur ou redresseur de soudage. Ce sont des appareils mixtes ( $A C-D C$ transformer-rectifier power source; $A C$-DC power source). Lorsque l'on soude en courant alternatif, seule la partie transformateur de la source de courant est utilisée ; en courant continu, le transformateur et le redresseur sont tous deux mis à contribution.

c) Autres critères de classification des postes à soudage à l'arc (figure 2) 
Figure 2

APPAREIL À COURANT CONSTANT ;

appareil de soudage à

appareil de soudage à

caractéristique tombante; caractéristique plongeante

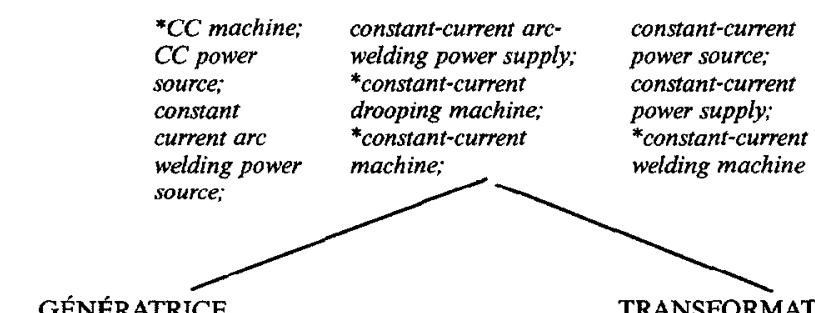

GÉNÉRATRICE

TRANSFORMATEUR-REDRESSEUR DE COURANT CONSTANT génératrice

à caractéristique tombante constant-current welding generator

À CARACTÉRISTIQUE TOMBANTE rectifier-type power source

(Par analogie avec "constant-voltage rectifier type power source')

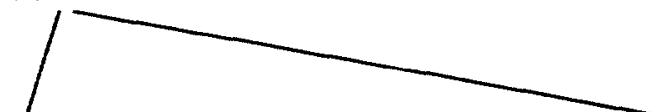

GÉNÉRATRICE

DE COURANT CONSTANT

ENTRAINÉE PAR MOTEUR ÉLECTRIQUE

constant-current motor generator
GÉNÉRATRICE

DE COURANT CONSTANT

ENTRAÎNÉE PAR MOTEUR THERMIOUE

(terme non attesté en anglais)
APPAREIL

\section{À TENSION CONSTANTE:}

appareil

à caractéristique plate;

poste

à caractéristique horizontale

constant potential

power source;

constant potential

unit:

constant-voltage

arc-welding

power supply;

constant voltage

power source

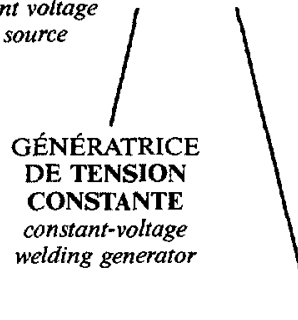

poste

à potentiel constant; poste

à tension constante

constant-voltage

power supply;

constant-voltage

supply;

constant-voltage

unit;

$C P$ unit:

$C V$ power source

CV unit
TRANSFORMATEUR-REDRESSEUR

A CARACTERISTIQUE

PLATE

PLATE
constant-voltage

rectifier-type power source
APPAREIL

À CARACTÉRISTIQUE MONTANTE

*rising voltage

arc welding machine;

*rising voltage

*rising-voltage

machine:

machine;

rising-voltage

power source

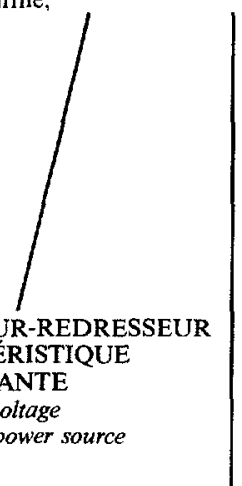

GÉNÉRATRICE

À CARACTÉRISTIQUE MONTANTE

rising-voltage

welding generator 
Comme nous l'avons expliqué au début de ce chapitre, il existe plusieurs façons de classer les postes de soudage à l'arc. Même si nous avons opté pour celle qui se fonde sur le type de courant produit par l'appareil (alternatif ou continu), on ne peut passer sous silence celle qui se base sur les caractéristiques du courant produit par l'appareil. En effet, trois sources de courant importantes, appartenant aux appareils à courant continu, ont été nommées d'après ce mode de classification. Ainsi, on trouve les appareils à courant constant ou appareils de soudage à caractéristique tombante ou plongeante (constant-current power source; constant-current arc-welding power supply ${ }^{11}$ ), les appareils à tension constante ou appareils à caractéristique plate ${ }^{12}$ (constant-voltage power source ; constant-voltage arc-welding power supply ${ }^{13}$ ) et les appareils à caractéristique montante (rising-voltage power source ${ }^{14}$ ). Les termes "caractéristique tombante" (voir figure 3a), "caractéristique plate " (voir figure $3 \mathrm{~b}$ ) et "caractéristique montante » (voir figure 3c) décrivent la relation entre la tension et l'intensité générées par la source de courant et utilisées pour le soudage. Il dépasserait le cadre de ce travail de tenter d'exposer les principes et le fonctionnement des trois appareils mentionnés plus haut. Nous préférons renvoyer les lecteurs ayant de bonnes connaissances en électricité et en physique à des articles spécialisés, par exemple celui de Roland Cazes dans les Techniques de l'ingénieur. Pour les besoins de cet exposé, il suffit de savoir que chaque poste de soudage est utilisé pour mettre en cuvre des procédés particuliers. Ainsi, les appareils à courant constant sont utilisés principalement pour le soudage à l'arc avec électrode enrobée et le soudage TIG, parce qu'un courant constant est nécessaire pour le bon fonctionnement de ces procédés. Les appareils à tension constante sont utilisés pour le soudage MIG. La tension de ce type de source de courant demeure relativement constante sur toute la gamme normale des courants utilisés. C'est une caractéristique essentielle à ce type de procédé. Les appareils à caractéristique montante sont peu utilisés car la tendance est de se servir des appareils à tension constante. Cependant, cette source de courant est conçue pour les procédés automatisés, comme le soudage GMAW à très grande vitesse.

Ces trois postes de soudage ne diffèrent pas, du point de vue technologique, des autres grandes sous-catégories d'appareils de soudage (figure 1). On trouve ainsi, d'une part, des génératrices de courant constant ou génératrices à caractéristique tombante (principe rotatif) [(termes non attestés en français, construits par analogie avec "génératrice de courant alternatif ", "génératrice de courant continu " et " appareil à caractéristique tombante ») (constant-current welding generator)] entraînées par moteur électrique (constant-current motor generator) ou par moteur thermique (terme non attesté en anglais) et, d'autre part, des transformateurs-redresseurs à caractéristique tombante (principe statique) (termes non attestés ni en anglais ni en français, créés par analogie avec le terme anglais "constant-voltage rectifier-type power source" et avec le terme français « appareil à caractéristique montante ») (voir figure 2 pour d'autres sous-types).

La puissance électrique fournie par les appareils et les conditions d'utilisation de ces derniers constituent d'autres critères permettant de classer les appareils. Les sources documentaires consultées ne nous ont pas permis d'effectuer un recoupement notionnel bilingue. Nous avons cependant cru bon d'inclure les renseignements suivants.

La puissance électrique fournie par les appareils de soudage

Selon Menar 1970, les postes de soudage peuvent se diviser en trois groupes :

a) Les postes légers $(\mathrm{I}<150 \mathrm{~A})$

b) Les postes normaux (I $<250$ ou $300 \mathrm{~A}$ )

c) Les postes à grande puissance (I $>600 \mathrm{~A})$

Whand et Weldi découpent la réalité de façon différente. On trouve ainsi les « limited input power source" ou "limited-service power source" $(\mathrm{I}<300 \mathrm{~A})$ et les " indus- 
Figure 3

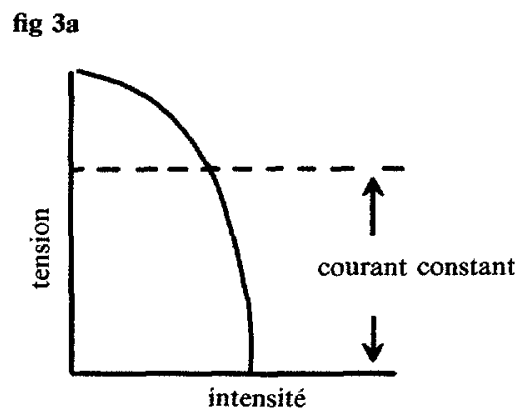

SMIWE 84284

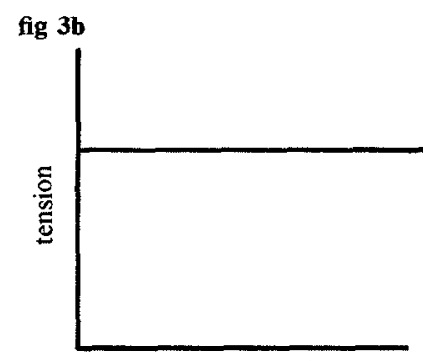

intensité

SMIWE 84285

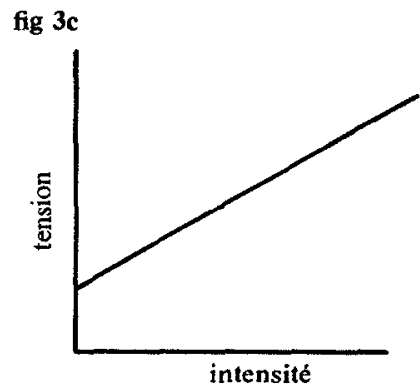

SMIWE 84434 
trial class welding power source » ou « industrial class power source » (facteur de marche de $60 \%$ ou plus).

Les conditions d'utilisation des appareils de soudage

Les appareils portatifs [(portable power source) (terme non attesté en anglais, construit par analogie avec "portable arc-welding machine ", terme à proscrire pour les mêmes raisons qu'en 2)] pèsent de 60 à $100 \mathrm{~kg}$ environ. Ils possèdent des poignées ou des brancards, et parfois des crochets d'élingage. Ces appareils sont destinés à des travaux légers.

Les appareils transportables sont montés sur des chariots (trailer) et sont trainés par un ou deux brancards. Le poids de ces appareils varie entre 100 et $600 \mathrm{~kg}$.

Les postes fixes sont soit installés à demeure dans l'atelier, soit montés sur wagon, camion ou remorque. Leur poids dépasse 600 à $700 \mathrm{~kg}$. (D'après Menar 1970 : 30.)

\section{LES APPAREILS DE SOUDAGE SPÉCIALISÉS}

Il existe des postes de soudage créés expressément pour répondre aux exigences d'un procédé spécifique. Ces appareils portent d'ailleurs le nom du procédé pour lequel ils ont été conçus. Ils ne diffèrent pas radicalement des postes de soudage déjà décrits, mais comportent néanmoins certaines particularités qui les distinguent de ces derniers. Ainsi, le poste de soudage TIG, encore appelé appareil de soudage TIG, source de courant TIG ou appareil TIG (TIG power source ${ }^{15}$ ), comporte, s'il s'agit d'un appareil TIG à courant alternatif, un circuit haute fréquence (high-frequency circuit) destiné à réamorcer l'arc au début de chaque alternance. Les appareils conçus spécialement pour le soudage TIG coûtent donc plus cher que les appareils non spécialisés pouvant également être utilisés pour ce procédé. Les postes de soudage au plasma [(terme non attesté en français, construit par analogie avec " TIG power source = poste de soudage TIG » et "plasma welding = soudage au plasma") (plasma-welding power source; plasma-arc power supply ${ }^{16}$ )] comportent plus de relais électriques que les appareils de soudage à courant continu de façon à produire une tension et un courant plus élevés. Les appareils de soudage MIG ou postes de soudage MIG, encore appelés appareils MIG (MIG power source ${ }^{17}$ ), sont créés pour fonctionner sans interruption, à la différence d'autres appareils qui ne peuvent être utilisés que pendant un temps prédéterminé.

\section{LES CARACTÉRISTIQUES COMMUNES AUX DIFFÉRENTS POSTES DE SOUDAGE À L'ARC}

Nous avons insisté jusqu'à maintenant sur les caractéristiques qui différencient les appareils de soudage les uns des autres. Les postes de soudage ont cependant des points communs, tant sur le plan abstrait que concret.

\section{a) Plan abstrait}

Tous les types d'appareils de soudage possèdent certaines caractéristiques abstraites qui permettent de les comparer entre eux. Parmi ces dernières, les plus importantes sont : la tension à vide (open-circuit voltage; no-load voltage; $O C V$ ), le facteur de marche (duty cycle) et le courant maximal de soudage (maximum amperage).

La tension à vide est la tension aux bornes (output terminal) de l'appareil de soudage lorsque le circuit extérieur est ouvert. Selon la National Electrical Manufacturers Association (NEMA), la tension à vide ne devrait pas dépasser 80 volts dans le cas du soudage manuel et 100 volts dans les procédés de soudage automatique et semiautomatique.

Contrairement aux autres appareils électriques qui doivent fournir du courant jusqu'au moment où l'on coupe ce dernier, les appareils de soudage ne fournissent de 
l'énergie que durant une période limitée, sauf si les appareils sont conçus expressément pour le soudage automatique. Le facteur de marche exprime, sous forme de pourcentage, le temps pendant lequel l'appareil doit fournir le courant de soudage au cours d'une période de dix minutes. Ainsi un facteur de marche de $60 \%$ (60\% duty cycle) signifie que l'appareil ne peut fournir du courant que pendant six minutes sur dix. Un facteur de marche de $100 \%$ signifie que le temps de marche à vide est nul.

\section{b) Plan concret}

Les postes de soudage se présentent sous plusieurs formes différentes. Néanmoins, ils sont toujours entièrement capotés de façon à protéger les organes internes de l'appareil. Sur le tableau de commande (control panel), on trouve des appareils de mesure, tels qu'un ampèremètre (ammeter) et un voltmètre (voltmeter). Ces cadrans indiquent au soudeur l'intensité du courant fourni par l'appareil et la tension à laquelle il est débité. Avant de mettre l'appareil en marche, le soudeur doit normalement consulter la plaque signalétique (name plate; nameplate) située à l'avant de l'appareil de soudage. Cette plaque a pour objet de faire connaître à l'utilisateur les caractéristiques électriques indispensables qui lui permettent d'en faire le meilleur usage. On y retrouve notamment la catégorie de l'appareil (transformateur de soudage, transformateur-redresseur de soudage, générateur, etc.), la nature de la caractéristique statique voltage-ampérage, la nature du courant de soudage, la tension à vide, la gamme de réglage de la machine, le facteur de marche, etc. Après avoir réglé l'intensité du courant (set the current, to) à l'aide d'un levier, volant (handwheel) ou commutateur, le soudeur met l'appareil en marche en appuyant sur le bouton marche (on button) situé également sur le tableau de commande.

Notes linguistiques

* Voir META, 31:4, pp. 407-420

1. Dans les ouvrages dépouillés, les auteurs francophones et anglophones posent comme synonymes les termes " poste de soudage " (power source) et "poste de soudage à l'arc" (arc welding power source). Selon nous, ce serait commettre la même erreur que d'affirmer que " procédé de soudage " est un synonyme de "procédé de soudage à l'are " que de poser en synonymes les termes cités plus haut. Tout comme il existe différents types de procédés de soudage, il existe également différents types de postes de soudage, le poste de soudage à l'arc en étant un. Ces deux termes ne se situent pas au même niveau hiérarchique dans l'arbre du domaine, le poste de soudage étant un générique par rapport au poste de soudage à l'arc. Il est donc faux de poser comme synonymes les termes "poste de soudage" et "poste de soudage à l'arc". Le synonyme " source de courant " ne devrait être utilisé qu'en contexte étant donné son caractère très général. Même remarque pour les synonymes anglais de "welding power source", soit "power source " et "power supply".

2. Les termes welding machine et welder sont à proscrire comme synonymes du générique "welding power source ". Cette recommandation s'appuie sur l'extrait suivant d'une norme éditée par l'American Welding Society.

The terms "welder" and "welding operator" are applicable to the individual only, and the machine performing the welding operation should be referred to as the "welding machine".

The equipment that supplies current for electric welding should be referred to as either a "welding generator" or a "welding transformer" (...) (Whand $1976: 28.282$ )

3. Les termes arc welder (Grasc 1982:672), arc welding machine (Pewel $1968: 169$ ) sont à proscrire comme synonymes du spécifique "arc-welding power source" pour les mêmes raisons qu'en 2.

4. Egalement : $A$-C power source (Weldi 1968 : 67); alternating-current welding power source (Whand 1968 : 25.41); alternating-current power source (Whand $1968: 25.12$ ) ; A-C welding power source (Weldi 1968 : 67); $A$-C power supply (Whand $1968: 25.54$ ). Les synonymes $A C$ machine (Smiwe $1984: 274) ; A C$ arc welding machine (Amode $1980: 213$ ) ; AC arc-welding machine (Smiwe $1984: 294$ ) ; alternating current machine (Pewel 1968: 100); alternating current welding machine (Weldi 1968:66); A-C welding machine (Whand $1968: 25.11$ ) sont à proscrire pour les mêmes raisons qu'en 2 .

5. Dans $90 \%$ des cas, le « transformateur » est alimenté en courant alternatif. Toutefois, dans le domaine du soudage, nous ne croyons pas qu'il soit redondant de préciser le type de courant transformé pour que l'on 
puisse bien différencier le "transformateur de courant alternatif" du "transformateur de courant continu " utilisé dans les appareils à courant continu (Smiwe $1984: 275$ ).

6. Également : alternating current arc welding transformer (Amode $1980: 229$ ) ; alternating-current transformer (Whand $1968: 25.22$ ) ; A-C welding transformer (Weldi $1968: 78$ ) ; AC transformer power source (Smiwe 1984 : 325); alternating-current transformer power source (Whand 1968 : 25.19). À proscrire : $A-C$ transformer welding machine (Weldi $1968: 75$ ) pour les mêmes raisons qu'en 2.

7. Synonyme à proscrire : engine driven $A C$ arc welder (Amode $1980: 216$ ) pour les mêmes raisons qu'en 2.

8. Également : direct current power supply (Whand $1968: 25.54$ );D-C power source (Weldi $1968: 147$ ). A proscrire : DC machine (Pewel 1965:100); DC welding machine (Smiwe $1984: 279$ ) ; DC welder (Grasc 1982 : 1-671); direct current machine (Pewel 1968: 100); direct-current welder (Grasc $1982: 672$ ) ; DC arc welding machine (Amode $1980: 157$ ) ; DC arc welder (Amode $1980: 157$ ) pour les mêmes raisons qu'en 2.

9. Egalement : transformer-rectifier D-C power source (Weldi $1968: 65$ ); transformer-rectifier power source (Weldi $1968: 167$ ) ; DC rectifier power source (Smiwe $1984: 279$ ).

A proscrire : *transformer-rectifier machine (Smiwe $1984: 325$ ); *transformer-rectifier D-C welding machine (Weldi $1968: 69$ ) pour les mêmes raisons qu'en 2 .

10. À proscrire : ${ }^{*} D C$ motor-generator welding machine (Amode $1980: 184$ ); ${ }^{*} D C$ motor generator arc welder (Amode $1980: 187$ ) pour les mêmes raisons qu'en 2.

11. Également : drooper (Smiwe 1984:282); CC power source (Smiwe $1984: 319$ ); constant-current power supply (Whand $1968: 25.4$ ); constant-current arc welding power source (Whand $1968: 25.6$ ). À proscrire: *constant-current drooper machine (Smiwe $1984: 282$ ) ; *constant-current machine (Smiwe $1984: 282$ ) ; *CC machine (Smiwe $1984: 284$ ) ; * constant-current welding machine (Smiwe $1984: 284$ ) pour les mêmes raisons qu'en 2.

12. Également : poste à tension constante (Dusou $1971: 226$ ); poste à caractéristique horizontale (Dusou $1971: 226$ ) ; poste à potentiel constant (d'après Cagen : 5).

13. Également : constant potential unit (Smiwe 1984: 284); CP unit (Smiwe $1984: 284$ ); CV power source (Smiwe 1984 : 284) ; constant-voltage unit (Smiwe $1984: 284$ ) ; CV unit (Smiwe 1984 : 284) ; constantvoltage supply (Whand $1968: 25.4$ ); constant voltage power source (Smiwe $1984: 285$ ) ; constant-voltage power supply (Whand $1968: 25.7$ ); constant potential power source (Weldi 1968 : 188).

14. À proscrire pour les mêmes raisons qu'en 2 : *rising-voltage machine (Smiwe $1984: 436$ ); ${ }^{*}$ rising voltage machine (Amode $1980: 299) ;{ }^{*}$ rising voltage arc welding machine (Amode $1980: 299$ ).

15. À proscrire pour les mêmes raisons qu'en $2:{ }^{*}$ heliwelder welding machine (Smiwe $1984: 316$ ) ; *T.I.G. welding machine (Pewel 1968 : 181).

16. Également: Paw power source (Smiwe $1984: 351$ ); plasma-arc power source (Smiwe 1984 : 352); plasma power source (Smiwe 1984 : 352); plasma arc-welding power source (Smiwe $1984: 293$ ) ; plasma power supply (Smiwe 1984 : 351). À proscrire : *plasma-arc machine (Smiwe $1984: 353$ ); *plasma machine (Smiwe $1984: 293$ ) pour les mêmes raisons qu'en 2.

17. Â proscrire: *MIG welding machine (Smiwe $1984: 284$ ) ; *M.I.G. welding machine (Pewel $1968: 181$ ) pour les mêmes raisons qu'en 2 . 